\title{
Flux Renormalization in Constant Power Burnup Calculations
}

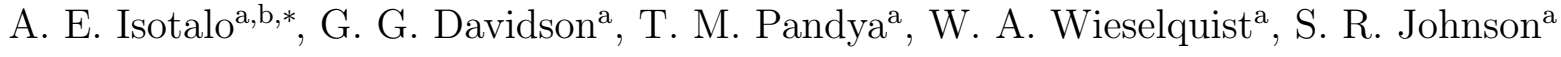 \\ ${ }^{a}$ Oak Ridge National Laboratory, PO Box 2008, Oak Ridge, TN 37831, USA \\ ${ }^{b}$ Aalto University, PO Box 14100, FI-00076 AALTO, Finland
}

\begin{abstract}
To more accurately represent the desired power in a constant power burnup calculation, the depletion steps of the calculation can be divided into substeps and the neutron flux renormalized on each substep to match the desired power. This paper explores how such renormalization should be performed, how large a difference it makes, and whether using renormalization affects results regarding the relative performance of different neutronics-depletion coupling schemes. When used with older coupling schemes, renormalization can provide a considerable improvement in overall accuracy. With previously published higher order coupling schemes, which are more accurate to begin with, renormalization has a much smaller effect. While renormalization narrows the differences in the accuracies of different coupling schemes, their order of accuracy is not affected.
\end{abstract}

Keywords: burnup calculations, renormalization, substeps, constant power depletion

\section{Introduction}

Burnup calculations simulate changes in the nuclide composition and neutronics performance of nuclear fuel under irradiation. The composition of the fuel affects the neutron spectrum, which in turn affects the reaction rates and hence the composition. Due to computational limitations, this coupled problem between transport and depletion is usually solved as a sequence of steady state transport calculations and constant flux depletion steps. Transport calculations driven by a criticality source only produce unnormalized fluxes, so burnup calculations for nuclear reactors also require a normalization condition that specifies the magnitude of the flux.

Most burnup calculations are normalized so that the modeled system generates a specific amount of power. The power may differ from step to step, but it is usually constant during any given step, which is also assumed to be the case in this paper.

${ }^{*}$ Corresponding author

Email address: aarno.isotalo@aalto.fi (A. E. Isotalo)

This manuscript has been authored by UT-Battelle, LLC under Contract No. DE-AC05-00OR22725 with the U.S. Department of Energy. The United States Government retains and the publisher, by accepting the article for publication, acknowledges that the United States Government retains a non-exclusive, paid-up, irrevocable, world-wide license to publish or reproduce the published form of this manuscript, or allow others to do so, for United States Government purposes. The Department of Energy will provide public access to these results of federally sponsored research in accordance with the DOE Public Access Plan (http://energy.gov/downloads/doepublic-access-plan) 
The power is assumed to be known at all times, and it specifies the magnitude of the flux. However, depletion calculations, which actually progress the time and burnup in the simulation, require flux rather than power to remain constant. At each step, the power must thus be converted to a flux that gives the correct step average power. Power-to-flux conversion is exact for given cross sections, library data, and material compositions. However, calculating a step-average flux that would produce the desired average power is always approximate, as the fuel composition will change during the step and these changes are not known in advance. Because of this, the flux used for depletion will never reproduce the desired power exactly, and the calculated material compositions thus correspond to a different burnup than the user-specified power implies. We call this difference in burnup the normalization error. Furthermore, regardless of how accurate the step average normalization is, additional error arises from the flux being forced constant when it should be continuously changing to keep the power constant.

These errors result from time discretization and can be reduced by using shorter steps. Another way to reduce the errors is to divide each depletion step into substeps and renormalize the flux on each substep without performing additional transport calculations. In addition to making the step average normalization more accurate, this procedure allows the flux to change in a piecewise constant manner during the step, which reduces the error that would be caused by keeping it constant throughout the entire step. It is also possible to perform renormalization without using substeps, i.e., with only one substep, in which case only the step average normalization is affected.

This paper explores three questions related to renormalization in constant power burnup calculations: how should it be performed, how much of a difference does it make, and does considering it affect the conclusions of Isotalo and Sahlberg (2015) and Isotalo (2015a) regarding the advantage of the higher order neutronics-depletion coupling schemes (Isotalo and Aarnio, 2011a,b) over older methods.

\section{Neutronics-depletion coupling}

The coupling of sequential transport solutions and depletion steps into a burnup calculation can be accomplished in numerous ways, even when renormalization options are not considered. The different methods used for this are called coupling schemes. While renormalization is part of the coupling, it is considered separate from the actual coupling scheme in this paper.

The effects of renormalization are tested for four different coupling schemes. Lacking unambiguous or established names, the methods are labeled using the schematic representation of Isotalo and Aarnio (2011a) and Isotalo and Sahlberg (2015). Note that while the labels have been formed as abbreviations, the abbreviated words are not intended to form unambiguous names for the methods. Two of the included methods; LE, from "linear extrapolation", and LE/QI, from "linear extrapolation / quadratic interpolation", are higher order schemes that use substeps even when not renormalizing (Isotalo and Aarnio, 2011a,b). The other two coupling schemes are different midstep methods labeled as CE/CM, from "constant extrapolation / constant midpoint", and Ce/CM, from "constant extrapolation (of previous step values) / constant midpoint". Brief descriptions of the coupling schemes without renormalization are provided below. How renormalization changes the coupling is discussed in Section 3. 


\section{1. $C E / C M$}

On the predictor of $\mathrm{CE} / \mathrm{CM}$, transport is performed with beginning-of-step compositions. The obtained cross sections and neutron fluxes are then used to deplete the materials for half of the desired step length. On the corrector, transport is performed with the middle-of-step compositions, and the beginning-of-step compositions are depleted for the full step length using the middle-of-step cross sections and fluxes. The obtained end-of-step compositions become the initial compositions for the next step. This coupling scheme is used, for example, in MCNP6 (Goorley et al., 2012).

\section{2. $C e / C M$}

On the predictor of $\mathrm{Ce} / \mathrm{CM}$, the cross sections and fluxes from the middle of the previous step are used to deplete the beginning-of-step compositions for half of the desired step length. On the corrector, transport is performed with the middle-of-step compositions, and the beginning-ofstep compositions are depleted for the full step length using the middle-of-step cross sections and fluxes. The obtained end-of-step compositions become the initial compositions for the next step. On the first step, an additional transport calculation is performed with the initial compositions and the obtained results used in place of the previous step values. What differentiates Ce/CM from $\mathrm{CE} / \mathrm{CM}$ is that after the first step, there is no transport solution on the predictor. Ce/CM is used, for example, in TRITON (Jessee and DeHart, 2011).

\section{3. $L E$ and $L E / Q I$}

In LE, transport is first performed with the beginning-of-step compositions. Then each individual cross section and local flux is linearly extrapolated through the beginning-of-the-previousstep and beginning-of-the-current-step values. The step is divided into equidistant substeps, and on each substep, substep average cross sections and fluxes from the linear extrapolation are used for depletion. The obtained end-of-step compositions become the initial compositions for the next step. There is no corrector.

LE/QI starts with LE as a predictor. This is followed by a corrector where transport is performed with the predicted end-of-step compositions. A quadratic interpolation is then made using the previous step, beginning-of-step, and end-of-step values for each cross section and flux. The materials are re-depleted with the substep averages coming from the quadratic interpolation, and the new end-of-step compositions become the initial compositions for the next step.

On the first step, linear extrapolation and quadratic interpolation are replaced by constant extrapolation and linear interpolation, respectively. Quadratic interpolation is also replaced with linear interpolation on the second step. These higher order methods are used in Serpent 2 (Leppänen, 2015) and in Shift (Pandya et al., 2015), in which the internal burnup calculation capability is currently being finalized. Note that while LE/LI (Isotalo and Aarnio, 2011a,b) was

not included in this paper, it is still considered relevant and might be preferable over the included methods.

\subsection{Normalization}

The total power of the modeled system can be calculated as

$$
P(t)=P_{\mathrm{d}}(t)+P_{\mathrm{r}}(t)=\sum_{i} \sum_{j} V_{i} n_{i, j}(t) \lambda_{j} \kappa_{j, \mathrm{~d}}+\sum_{i} \sum_{j} \sum_{r} V_{i} n_{i, j}(t) \kappa_{j, r}(t) \sigma_{i, j, r}(t) \phi_{i}(t),
$$

where 
$P_{\mathrm{d}}(t)$ is the power generated through radioactive decay at time $t$,

$P_{\mathrm{r}}(t)$ is the power generated by neutron-induced reactions at time $t$,

$V_{i}$ is the volume of material region $i$,

$n_{i, j}(t)$ is the average atomic density of nuclide $j$ in region $i$ at time $t$,

$\lambda_{j}$ is the decay constant of nuclide $j$,

$\kappa_{j, \mathrm{~d}}$ is the average recoverable energy released in the decay of nuclide $j$,

$\phi_{i}(t)$ is the homogenized one-group flux in region $i$ at time $t$,

$\sigma_{i, j, r}(t)$ is the homogenized one-group cross section of reaction $r$ of nuclide $j$ in region $i$ at time $t$, and

$\kappa_{j, r}(t)$ is the expected average recoverable energy released in reaction $r$ of nuclide $j$ at time

$t$.

In constant power burnup calculations, the power $P(t)=P$ is input, and the fluxes used in depletion should reproduce it. The fluxes produced by transport calculations for critical systems are unnormalized and differ from the desired ones by some constant. They can be normalized to produce the desired power as

$$
\phi_{i}(t)=\frac{P-P_{\mathrm{d}}(t)}{P_{\mathrm{r}}^{*}(t)} \phi_{i}^{*}(t),
$$

where $P_{\mathrm{r}}^{*}(t)$ is the reaction power calculated with the unnormalized fluxes $\phi_{i}^{*}(t)$. This is generally done immediately after transport and may be considered a part of the transport solution itself, which was also assumed to be the case in the above descriptions of the coupling schemes.

Due to data limitations and other issues, the energy production is often approximated by setting $\kappa_{j, \mathrm{~d}}=0$ and approximately including the energy released in decay reactions into the energy released in the preceding neutron induced reactions. This approximation is also done in the library data used for this work, although the codes are capable of handling decay energy explicitly. The energy generation may also be further approximated by only having energy explicitly produced in fissions. Neither of these approximations fundamentally alters the normalization.

\section{Renormalization methods}

As the nuclide concentrations change over time, the normalization performed after transport is only instantaneously accurate. Renormalization aims to make the normalization more representative of the step as a whole. This is usually combined with substeps, so that the normalization only needs to be fixed for a short period at a time.

There are multiple ways in which renormalization can be done. In this work, the included methods are referred to as beginning-of-substep renormalization (labeled as BOSS in figures and tables), middle-of-substep renormalization (labeled as MOSS), energy-based renormalization (labeled as ENE), and ORIGEN-style renormalization (labeled as ORIGEN). In addition, using no renormalization is labeled as NONE. The number of substeps used will follow the label. These four methods considered in this work are described in Sections 3.1-3.4. This list is not intended to be all-inclusive, and it is easy to come up with additional ways for performing renormalization. Some might even be in use. The described methods have been implemented into Shift, but at the time of writing this Shift has not yet been released.

As the true time dependence of cross-sections and flux is not known, $\kappa_{j, r}(t), \sigma_{i, j, r}(t)$, and $\phi_{i}^{*}(t)$ are approximated by whatever the neutronics-depletion coupling scheme predicts. For most coupling schemes, for example $\mathrm{CE} / \mathrm{CM}$ and $\mathrm{Ce} / \mathrm{CM}$, these predictions are simply whatever constant 
values were obtained in the latest transport solution. When this is the case, the beginningof-substep and substep-average values in the descriptions below simply become the constants assumed for that step. We are not aware of renormalization having previously been used with coupling schemes that do not make this assumption. The only exceptions considered in this work are LE and LE/QI, which do make time-dependent predictions.

Note that the flux predicted by the coupling scheme must already be normalized before the substep and renormalization process. The initial normalization (Eq. 2) after each transport calculation is thus required even when using renormalization. There are certain exceptions to this for some combinations of coupling scheme and renormalization method, but they have limited significance and will not be discussed here.

\subsection{Beginning-of-substep renormalization}

Beginning-of-substep renormalization is the simplest way to perform renormalization. On each substep, the beginning-of-substep cross sections and compositions are used to normalize the substep average fluxes as

$$
\phi_{i}(t)=\frac{P-P_{\mathrm{d}}(t)}{P_{\mathrm{r}}^{*}} \frac{1}{t_{1}-t_{0}} \int_{t_{0}}^{t_{1}} \phi_{i}^{*}(s) d s
$$

where

$$
P_{\mathrm{r}}^{*}=\sum_{i} \sum_{j} \sum_{r} V_{i} n_{i, j}\left(t_{0}\right) \kappa_{j, r}\left(t_{0}\right) \sigma_{i, j, r}\left(t_{0}\right) \frac{1}{t_{1}-t_{0}} \int_{t_{0}}^{t_{1}} \phi_{i}^{*}(s),
$$

$\phi_{i}^{*}(s)$ is the flux predicted by the coupling scheme, $t_{0}$ is the time at the beginning of the substep, and $t_{1}$ is the time at the end of the substep. The renormalized fluxes are then used to deplete the materials over the substep. This method is implemented in the SCALE 6.2 (Rearden and Jessee, 2016) version of Polaris (Jessee et al., 2014) and in MPACT (Zhu et al., 2014).

\subsection{Middle-of-substep renormalization}

Another fairly straightforward approach is to use the middle-of-substep values. At each substep, the materials are first depleted for half of the substep length using the fluxes predicted by the coupling scheme. The flux is then renormalized using the middle-of-substep compositions, together with substep-average cross sections and flux distribution:

$$
\phi_{i}=\frac{P-P_{\mathrm{d}}\left(t_{1 / 2}\right)}{P_{\mathrm{r}}^{*}} \frac{1}{t_{1}-t_{0}} \int_{t_{0}}^{t_{1}} \phi_{i}^{*}(s) d s,
$$

where

$$
P_{\mathrm{r}}^{*}=\sum_{i} \sum_{j} \sum_{r} V_{i} n_{i, j}\left(t_{1 / 2}\right) \frac{1}{\left(t_{1}-t_{0}\right)^{3}} \int_{t_{0}}^{t_{1}} \kappa_{j, r}(s) d s \int_{t_{0}}^{t_{1}} \sigma_{i, j, r}(s) d s \int_{t_{0}}^{t_{1}} \phi_{i}^{*}(s) d s
$$

and $t_{1 / 2}=\left(t_{1}+t_{0}\right) / 2$ is the time at the middle the substep. The renormalized fluxes $\phi_{i}$ are then used to deplete for the full substep length. We are not aware of any prior use of this method, but in addition to Shift, it is now available in SCALE 6.2 Polaris. 


\subsection{Energy-based renormalization}

The ability to efficiently and accurately calculate time-integral results in depletion calculations (Isotalo, 2015b) makes it possible to base renormalization on the energy released during the (sub)step rather than the instantaneous power at some specific point in time. On each substep, a preliminary depletion calculation is performed for all materials with the substep average fluxes predicted by the coupling scheme. The energy released in these depletion calculations, $E_{\text {depl }}$, is compared to the amount of energy, $E=P t$, that should have been released based on the substep length, $t$, and the user-specified power, $P$. The flux in every material region is then multiplied by $E / E_{\text {depl }}$, and depletion is repeated using these new fluxes. This type of renormalization is made feasible by a recently developed capability and has not been used before.

\subsection{ORIGEN-style renormalization}

The point depletion code ORIGEN (Gauld, 2011) can be used with a different approach to renormalization. The power in each material region is assumed to remain constant at the coupling schemes predicted value over the entire step. This is a significant difference from the other renormalization methods discussed in this paper, which instead assume the flux distribution to be known.

The actual depletion of each material proceeds independently of the other materials. On each substep, a flux is first calculated based on the region power and the beginning-of-substep composition and cross sections as

$$
\phi_{i}\left(t_{0}\right)=\frac{P_{i}-\sum_{j} V_{i} n_{i, j}\left(t_{0}\right) \lambda_{j} \kappa_{j, \mathrm{~d}}\left(t_{0}\right)}{\sum_{j} \sum_{r} V_{i} n_{i, j}\left(t_{0}\right) \kappa_{j, r}\left(t_{0}\right) \sigma_{i, j, r}\left(t_{0}\right)},
$$

where $t_{0}$ is time at the beginning of the substep and $P_{i}$ is the power predicted for that region by the coupling scheme. This flux is then used to deplete the material for the length of the substep, after which an end-of-substep flux $\phi_{i}\left(t_{1}\right)$ is determined based on the obtained composition and the coupling-scheme-predicted end-of-substep values. This new flux is used to re-deplete the material, and the average of the compositions obtained with beginning-of-substep and end-ofsubstep fluxes becomes the final composition for the substep. This renormalization method is used by default in TRITON (Jessee and DeHart, 2011) and MCODE-3 (Gerrity, 2012), which use ORIGEN for depletion. This renormalization, or renormalization in general, is not used by all coupled codes that use ORIGEN as the depletion module.

\subsection{No renormalization}

It is always possible to only normalize the flux after solving transport and never renormalize. In this case the fluxes are used in the neutronics-depletion coupling in the same way as the cross sections. Most burnup calculation codes, for example CASMO-5 (Rhodes et al., 2007), MCNP6 (Goorley et al., 2012), VESTA (Haeck, 2012), ALEPH (Stankovskiy et al., 2010), BUCAL1 (El Bakkari et al., 2009), and Serpent (Leppänen, 2015), do not use renormalization. How accurately the normalization is maintained without renormalization depends on the neutronicsdepletion coupling scheme. How well the different coupling schemes maintain the normalization is one of the questions studied here. 


\section{Results}

Test calculations were performed using Shift (Pandya et al., 2015), which is currently being developed at ORNL, but at the time of writing this has not yet been officially released. The test case is a $2 \mathrm{D}$ segment of a Westinghouse $17 \times 17$ pressurized water reactor $(\mathrm{PWR})$ assembly with 248 regular fuel rods, 16 poison rods containing 6.0 wt. $\%$ of $\mathrm{Gd}_{2} \mathrm{O}_{3}$, and 25 empty guide tubes. The coolant water contains $760 \mathrm{ppm}$ of boric acid. Enrichment is 4.2 at.\% in the regular fuel rods and 2.7 at.\% in the gadolinium rods. The fuel in each normal fuel rod is treated as a single burnable region, while the poison rods are divided into ten concentric rings. Taking symmetries into account, there are 66 burnable material regions. This is the PWR test case used by Isotalo and Sahlberg (2015), who provide a more detailed description of the geometry.

The assembly is depleted with a constant specific power of $38.6 \mathrm{~kW} / \mathrm{kgHM}$ for 1600 days. All calculations used 20 inactive and 250 active cycles of 20000 neutrons, and the same ENDF/BVII.0 and JEFF3.0-A based SCALE 6.1 (Oak Ridge National Laboratory, 2011) nuclear data libraries. When analyzing the results, only the assembly average concentrations of the $53 \mathrm{nu}-$ clides listed in Table 1 were considered. This is the same set of nuclides that was used by Isotalo and Sahlberg (2015) and Isotalo (2015a), with ten additional fission products added based on significant macroscopic removal cross section in high burnup PWR fuel.

Each of the four coupling schemes described in Section 2 was combined with each of the four renormalization schemes described in Section 3, except that ORIGEN-style renormalization was not used with LE or LE/QI. ${ }^{1}$ No renormalization was considered as the fifth option. LE and $\mathrm{Ce} / \mathrm{CM}$, which require only one transport solution per step, used 32 steps, while CE/CM and LE/QI used 16 steps. Each step had 1, 2, 4, 8, or 16 substeps. In addition, a reference calculation was performed using LE/QI with 1024 steps and the middle-of-substep renormalization with 10 substeps.

The calculations with eight substeps and either energy-based renormalization or no renormalization were repeated 25 times. All other calculations, including the reference calculation, were repeated five times. The repeats were independent and identical apart from the sequence of random numbers used in the transport. Repeating the calculations reduces statistical variation in the final results, which are averages over the repeats. More importantly, the repeats allow the statistical uncertainty in the results to be quantified. The statistical uncertainties presented for the results are $95 \%$ confidence intervals that were calculated assuming the results to be normally distributed.

To ensure that the reference results are converged in respect to step lengths and hence contain no significant time discretization error, the reference calculation was also repeated with 512 steps. All differences between the results with 1024 and 512 steps were either statistically insignificant or at least two orders of magnitude below those between the reference calculations and the runs with 16 or 32 steps. The results presented for nuclide concentrations are the differences between the values obtained in the reference calculation with 1024 steps and in each of the other calculations. All error sources other than the time integration were identical in all calculations,

\footnotetext{
${ }^{1}$ The constant local powers in the ORIGEN-style renormalization conflict with the higher order coupling schemes which are partly based on predicting time-dependent flux and flux distribution. There are ways in which some of the aspects of ORIGEN-style renormalization could be used with the higher order coupling schemes, but these are not considered here.
} 
Table 1: The 53 nuclides considered when analyzing the results.

\begin{tabular}{|l|l|}
\hline Uranium & ${ }^{234} \mathrm{U},{ }^{235} \mathrm{U},{ }^{236} \mathrm{U},{ }^{237} \mathrm{U},{ }^{238} \mathrm{U},{ }^{239} \mathrm{U}$ \\
Plutonium & ${ }^{238} \mathrm{Pu},{ }^{239} \mathrm{Pu},{ }^{240} \mathrm{Pu},{ }^{241} \mathrm{Pu},{ }^{242} \mathrm{Pu}$ \\
Other actinides & ${ }^{239} \mathrm{~Np},{ }^{241} \mathrm{Am},{ }^{242 \mathrm{~m}} \mathrm{Am},{ }^{243} \mathrm{Am},{ }^{242} \mathrm{Cm},{ }^{244} \mathrm{Cm},{ }^{245} \mathrm{Cm}$ \\
Gadolinium & ${ }^{155} \mathrm{Gd},{ }^{157} \mathrm{Gd},{ }^{158} \mathrm{Gd}$ \\
Fission products & ${ }^{79} \mathrm{Se},{ }^{90} \mathrm{Sr},{ }^{90} \mathrm{Y},{ }^{93} \mathrm{Zr},{ }^{95} \mathrm{Mo},{ }^{99} \mathrm{Mo},{ }^{99} \mathrm{Tc},{ }^{101} \mathrm{Ru},{ }^{103} \mathrm{Rh},{ }^{105} \mathrm{~Pb},{ }^{109} \mathrm{Ag},{ }^{129} \mathrm{I}$, \\
& ${ }^{131} \mathrm{I},{ }^{131} \mathrm{Xe},{ }^{135} \mathrm{Xe}{ }^{133} \mathrm{Cs},{ }^{134} \mathrm{Cs},{ }^{135} \mathrm{Cs},{ }^{137} \mathrm{Cs},{ }^{137 m} \mathrm{Ba},{ }^{143} \mathrm{Nd},{ }^{145} \mathrm{Nd},{ }^{147} \mathrm{Pm}$, \\
\hline
\end{tabular}

so any differences in the results must be related to the time discretization, which has essentially been isolated from all other error sources. Because the reference results contain no significant time discretization error, the results thus directly show the time discretization error with each method.

Note that practical applications will always include other error sources, in particular those related to nuclear data and modeling assumptions, which may also cause errors attributable to the normalization. This work only considers errors related to the time discretization in the calculations. Furthermore, since we are dealing with time discretization errors specifically, they could always be reduced simply by reducing the step lengths. Any of the methods can be used to reach any level of accuracy in the time integration. The purpose of looking for the most accurate method is not to be able to obtain unprecedentedly accurate results, but to obtain accurate enough results with fewer steps and thus shorter computation time.

\subsection{Renormalization with $C E / C M$ and $C e / C M$}

Table 2 shows the maximum stepwise normalization errors ${ }^{2}$ when different renormalization strategies are used with $\mathrm{CE} / \mathrm{CM}$ and Ce/CM. Energy-based renormalization brings the error to practically zero, even without substeps. With a few substeps, middle-of-substep or ORIGEN-style renormalization also eliminates the error.

Beginning-of-substep renormalization, on the other hand, actually increases the normalization error unless the number of substeps is large. With eight or fewer substeps, the the atomic densities of most individual nuclides have larger errors than without renormalization. The errors decrease steadily with increasing number of substeps, and with 16 substeps, the effects are predominantly positive. With enough substeps, beginning-of-substep renormalization should be as accurate as the other methods. However, more than twice as many substeps are required, which negates any advantage the method might have from depleting the materials once on each substep. As beginning-of-substep renormalization is not competitive against other renormalization methods, it is not considered further.

\subsubsection{Short-lived nuclides}

Renormalization with substeps has a clear effect on short-lived nuclides (i.e., nuclides with effective half-life less than the step length), but differences in results obtained with the three

\footnotetext{
${ }^{2}$ Stepwise normalization error is the difference between the amount of energy released during the depletion calculations for a given step (Isotalo, 2015b) and the amount of energy that should have been released with the desired constant power.
} 
Table 2: Maximum stepwise normalization error (\%) with different renormalization strategies when using $\mathrm{CE} / \mathrm{CM}$ or $\mathrm{Ce} / \mathrm{CM}$.

\begin{tabular}{|c|c|c|c|c|c|}
\hline \multicolumn{6}{|c|}{$\mathrm{CE} / \mathrm{CM}$} \\
\hline \multirow{2}{*}{$\begin{array}{l}\text { Renormalization } \\
\text { method }\end{array}$} & \multicolumn{5}{|c|}{ Number of substeps } \\
\hline & 1 & 2 & 4 & 8 & 16 \\
\hline NONE & $-0.437 \pm 0.018$ & - & - & - & - \\
\hline BOSS & $-5.181 \pm 0.023$ & $-2.641 \pm 0.004$ & $-1.331 \pm 0.006$ & $-0.665 \pm 0.002$ & $-0.334 \pm 0.001$ \\
\hline MOSS & $0.212 \pm 0.001$ & $0.058 \pm 0.001$ & $0.015 \pm 0.001$ & $0.003 \pm 0.001$ & $-0.001 \pm 0.001$ \\
\hline ENE & $-0.016 \pm 0.001$ & $-0.005 \pm 0.001$ & $-0.003 \pm 0.001$ & $-0.003 \pm 0.001$ & $-0.002 \pm 0.001$ \\
\hline ORIGEN & $-0.467 \pm 0.003$ & $-0.136 \pm 0.001$ & $-0.040 \pm 0.001$ & $-0.013 \pm 0.001$ & $-0.004 \pm 0.001$ \\
\hline \multicolumn{6}{|c|}{$\mathrm{Ce} / \mathrm{CM}$} \\
\hline \multirow{2}{*}{$\begin{array}{c}\text { Renormalization } \\
\text { method }\end{array}$} & \multicolumn{5}{|c|}{ Number of substeps } \\
\hline & 1 & 2 & 4 & 8 & 16 \\
\hline NONE & $-0.325 \pm 0.010$ & - & - & - & \\
\hline BOSS & $-2.501 \pm 0.005$ & $-1.262 \pm 0.003$ & $-0.633 \pm 0.004$ & $-0.317 \pm 0.001$ & $-0.158 \pm 0.001$ \\
\hline MOSS & $0.048 \pm 0.001$ & $0.011 \pm 0.001$ & $0.002 \pm 0.001$ & $-0.001 \pm 0.001$ & $-0.001 \pm 0.001$ \\
\hline ENE & $-0.006 \pm 0.001$ & $-0.003 \pm 0.001$ & $-0.002 \pm 0.001$ & $-0.002 \pm 0.001$ & $-0.001 \pm 0.001$ \\
\hline ORIGEN & $-0.123 \pm 0.002$ & $-0.037 \pm 0.001$ & $-0.012 \pm 0.001$ & $-0.004 \pm 0.001$ & $-0.001 \pm 0.001$ \\
\hline
\end{tabular}
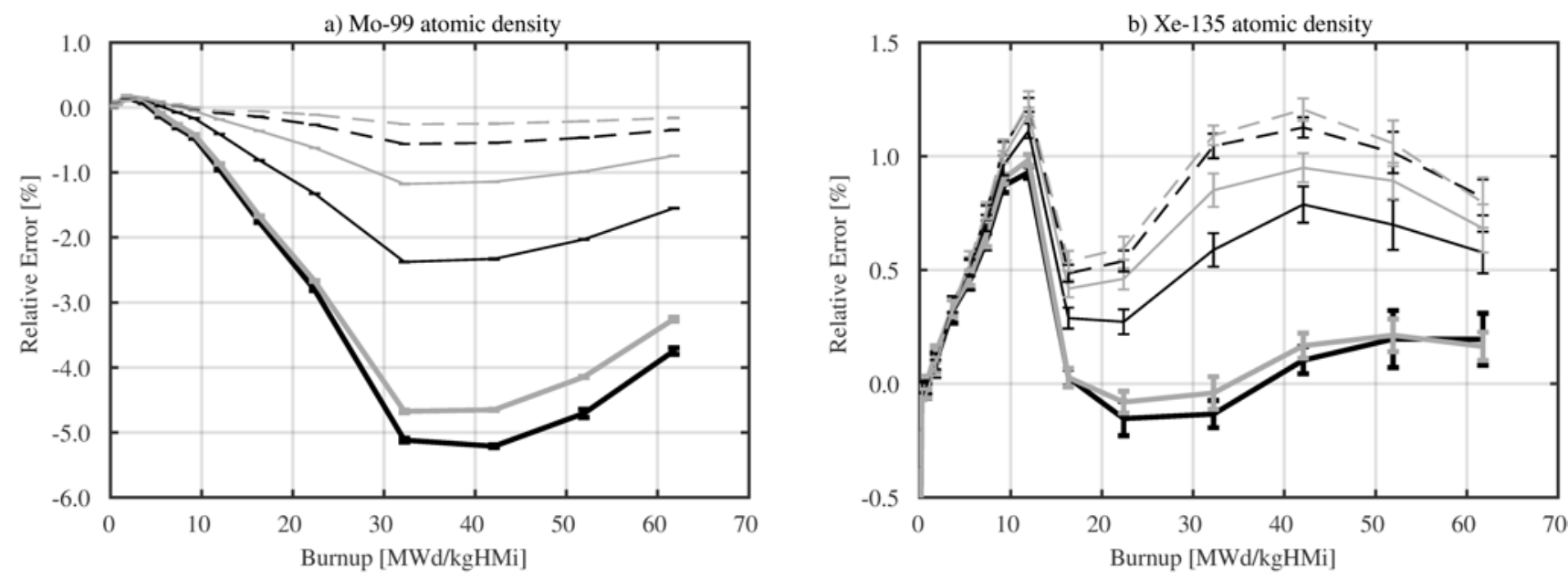

- NONE $\quad$ MOSS-1 $\longrightarrow$ MOSS-2 $\quad$ MOSS-4 --- MOSS-8 $\quad---$ MOSS-16

Figure 1: Relative errors in ${ }^{99} \mathrm{Mo}$ and ${ }^{135} \mathrm{Xe}$ atomic densities when using CE/CM with middle-ofsubstep renormalization and different numbers of substeps.

remaining renormalization methods are minimal. $\mathrm{CE} / \mathrm{CM}$ and $\mathrm{Ce} / \mathrm{CM}$ also produce qualitatively similar results for the short-lived nuclides, but with $\mathrm{Ce} / \mathrm{CM}$ the errors are only half as large as with $\mathrm{CE} / \mathrm{CM}$.

Figure 1 shows the relative errors in ${ }^{99} \mathrm{Mo}$ and ${ }^{135} \mathrm{Xe}$ atomic densities with $\mathrm{CE} / \mathrm{CM}$, middleof-substep renormalizations, and different numbers of substeps. Renormalization with a sufficient number of substeps greatly reduces the otherwise significant error in the atomic density of ${ }^{99} \mathrm{Mo}$, as well as similar errors for other short-lived nuclides that are not major absorbers. However, the accuracy for ${ }^{135} \mathrm{Xe}$ (Fig. 1b) is actually reduced by renormalization. Errors in the concentrations of ${ }^{149} \mathrm{Sm},{ }^{148 \mathrm{~m}} \mathrm{Pm}$, and ${ }^{151} \mathrm{Sm}$ are also increased by a minor amount. 


\subsubsection{Long-lived nuclides}

The concentrations of long-lived nuclides are dominated by the integrals of the reaction rates over multiple steps. Accuracy is thus determined mostly by the accuracy of the step-average predictions, including normalization (Table 2). With substeps, middle-of-substep renormalization, energy-based renormalization, and ORIGEN-style renormalization all make these errors minimal, and there are no significant differences between the results obtained with them. The only exception is that ORIGEN-style renormalization reduces the maximum absolute and relative errors for ${ }^{155} \mathrm{Gd}$ and ${ }^{157} \mathrm{Gd}$ by roughly a quarter when used with $\mathrm{CE} / \mathrm{CM}$ and by a third when used with $\mathrm{Ce} / \mathrm{CM}$. Other renormalization methods have almost no effect on the depletion of the initial gadolinium.

With just one substep, or to a lesser extent with two substeps, there are notable differences between the different renormalization strategies. In these cases, the main trend is that energy-based renormalization is more accurate or equally accurate when compared to the other renormalization methods. There are some exceptions, but they do not seem to follow any clear pattern. One such exception is ${ }^{235} \mathrm{U}$ (Fig. 2a), which by some coincidental cancellation of errors is depleted most accurately with middle-of-substep renormalization and one substep. Using more substeps or a different renormalization method leads to less accurate results.

Long-lived fission products, such as ${ }^{137} \mathrm{Cs}$ (Fig. 2b), whose concentrations mostly depend on how many fissions have occurred, show systematic improvement in line with the reducing normalization errors (Table 2). The errors in the concentrations of these nuclides are practically removed by renormalization with a few substeps, or by the energy-based renormalization even without substeps. However, as these errors are mostly below $0.3 \%$ without renormalization, eliminating them has only a limited significance, at least in this tested case with these step lengths.

With CE/CM, results for other uranium isotopes, as well as ${ }^{242} \mathrm{Pu},{ }^{241} \mathrm{Am}$ (Fig. 3a), and ${ }^{242 \mathrm{~m}} \mathrm{Am}$, also improve from renormalization, but plutonium isotopes other than ${ }^{242} \mathrm{Pu}$ show no significant change. Results for curium are mixed, and those for ${ }^{243} \mathrm{Am}$ (Fig. 3b) are less accurate with renormalization. Effects of renormalization with $\mathrm{Ce} / \mathrm{CM}$ are otherwise similar, but most errors are smaller, and the results for ${ }^{238} \mathrm{Pu},{ }^{240} \mathrm{Pu}$, and curium are slightly worse with renormalization than without. 

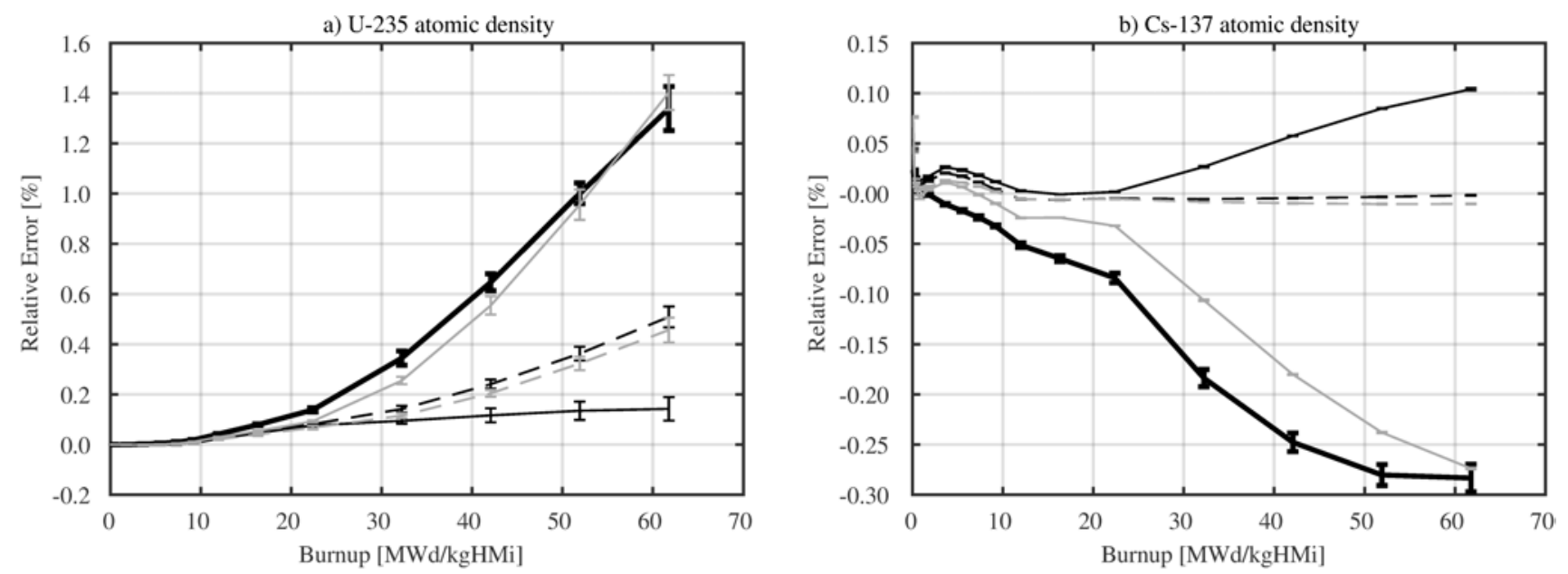

NONE

MOSS-1 $\quad---$ MOSS-8

ORIGEN-1 - - ORIGEN-8

Figure 2: Relative errors in ${ }^{235} \mathrm{U}$ and ${ }^{137} \mathrm{Cs}$ atomic densities when using $\mathrm{CE} / \mathrm{CM}$ with different renormalization strategies.
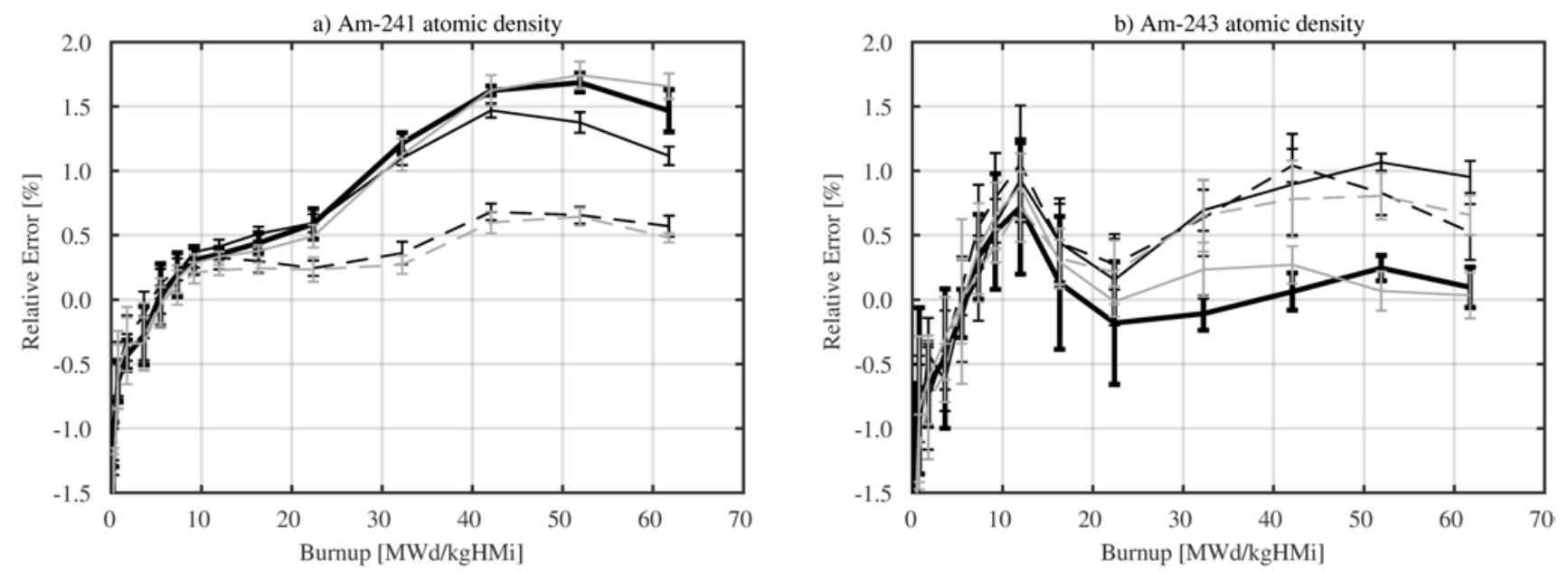

$$
\begin{array}{rlll|}
\hline \text { NONE } & \text { MOSS-1 } & --- \text { MOSS-8 } & \text { ORIGEN-1 }--- \text { ORIGEN-8 } \\
\hline
\end{array}
$$

Figure 3: Relative errors in ${ }^{241} \mathrm{Am}$ and ${ }^{243} \mathrm{Am}$ atomic densities when using $\mathrm{CE} / \mathrm{CM}$ with different renormalization strategies. 


\subsection{Renormalization with $L E / Q I$ and $L E$}

The higher order coupling schemes can use substeps without renormalization to update the cross sections and flux based on extrapolating or interpolating the values at the step boundaries. $\mathrm{LE} / \mathrm{QI}$ and LE are more accurate than $\mathrm{CE} / \mathrm{CM}$ and Ce/CM to begin with (Isotalo and Sahlberg, 2015; Isotalo, 2015a), and while using substeps provides them with significant benefits (Isotalo and Aarnio, 2011b), performing renormalization on those substeps has only a minor effect.

Table 3 shows the maximum stepwise normalization errors with LE/QI and LE. Compared with $\mathrm{CE} / \mathrm{CM}$ and $\mathrm{Ce} / \mathrm{CM}$ (Table 2), the most significant difference is that when renormalization is not used, the higher order coupling schemes maintain the normalization more accurately. This is especially true for LE/QI with substeps.

Errors in the atomic densities of long-lived fission products can again be reduced by renormalizing, but even without renormalization, the errors are below $0.10 \%$ with LE/QI and $0.15 \%$ with LE. Some other nuclides also show improvements of 0.1-0.2 percentage points (pp), while others have no significant errors to begin with or no significant change from renormalization. The largest effect from renormalization on substeps is that the error for ${ }^{99}$ Mo with LE decreases by almost $0.4 \mathrm{pp}$ on some steps. The loss of accuracy for short-lived poisons, which was observed when using renormalization with $\mathrm{CE} / \mathrm{CM}$ and Ce/CM, does not happen with LE or LE/QI.

When substeps are not used, renormalization has a larger effect than with eight substeps, but the effect is still smaller than with $\mathrm{CE} / \mathrm{CM}$ or $\mathrm{Ce} / \mathrm{CM}$, as the predicted step average fluxes are better to begin with. There were no notable differences between the middle-of-substep and energy-based renormalizations.

Table 3: Maximum stepwise normalization error (\%) with different renormalization strategies when using LE/QI or LE.

\begin{tabular}{|c|c|c|c|c|c|}
\hline \multicolumn{6}{|c|}{$\mathrm{LE} / \mathrm{QI}$} \\
\hline \multirow{2}{*}{$\begin{array}{c}\text { Renormalization } \\
\text { method }\end{array}$} & \multicolumn{5}{|c|}{ Number of substeps } \\
\hline & 1 & 2 & 4 & 8 & 16 \\
\hline NONE & $-0.274 \pm 0.012$ & $-0.107 \pm 0.008$ & $-0.075 \pm 0.005$ & $0.074 \pm 0.009$ & $0.078 \pm 0.007$ \\
\hline BOSS & $-4.337 \pm 0.019$ & $-2.220 \pm 0.019$ & $-1.120 \pm 0.003$ & $-0.560 \pm 0.002$ & $-0.280 \pm 0.002$ \\
\hline MOSS & $-0.042 \pm 0.007$ & $-0.020 \pm 0.001$ & $-0.006 \pm 0.001$ & $-0.002 \pm 0.001$ & $0.001 \pm 0.001$ \\
\hline ENE & $-0.013 \pm 0.001$ & $-0.003 \pm 0.001$ & $0.001 \pm 0.001$ & $0.001 \pm 0.001$ & $0.001 \pm 0.001$ \\
\hline \multicolumn{6}{|c|}{ LE } \\
\hline \multirow{2}{*}{$\begin{array}{c}\text { Renormalization } \\
\text { method }\end{array}$} & \multicolumn{5}{|c|}{ Number of substeps } \\
\hline & 1 & 2 & 4 & 8 & 16 \\
\hline NONE & $-0.220 \pm 0.014$ & $-0.206 \pm 0.010$ & $-0.190 \pm 0.006$ & $-0.191 \pm 0.007$ & $-0.190 \pm 0.006$ \\
\hline BOSS & $-2.161 \pm 0.030$ & $-1.112 \pm 0.012$ & $-0.559 \pm 0.009$ & $-0.278 \pm 0.005$ & $-0.141 \pm 0.002$ \\
\hline MOSS & $-0.023 \pm 0.003$ & $-0.009 \pm 0.001$ & $-0.003 \pm 0.001$ & $0.002 \pm 0.001$ & $0.001 \pm 0.001$ \\
\hline ENE & $-0.006 \pm 0.001$ & $-0.003 \pm 0.001$ & $0.002 \pm 0.001$ & $0.002 \pm 0.001$ & $0.001 \pm 0.001$ \\
\hline
\end{tabular}




\subsection{Comparison}

This section compares the results from $\mathrm{CE} / \mathrm{CM}$ and $\mathrm{Ce} / \mathrm{CM}$ with renormalization to LE/QI and LE with and without renormalization. Renormalization should generally be combined with substeps, and the higher order schemes should use substeps even when not renormalizing, so the results are only presented with eight substeps on each step.

Even without renormalization, LE/QI with substeps is very accurate for all fission products. With renormalization, $\mathrm{CE} / \mathrm{CM}$ and $\mathrm{Ce} / \mathrm{CM}$ can reach comparable accuracy for those that are not strong absorbers. However, differences in the results for ${ }^{135} \mathrm{Xe}$ and ${ }^{149} \mathrm{Sm}$ (Fig. 4) are actually larger than without renormalization due to the increased errors from Ce/CM and CE/CM. ${ }^{148 \mathrm{~m}} \mathrm{Pm}$ and ${ }^{151} \mathrm{Sm}$ also have significant differences in favor of LE/QI. LE is also clearly more accurate for the strong absorbers, even without renormalization, but it is slightly less accurate for the rest of the fission products unless renormalization is used.

Fig. 5 shows the errors in the atomic densities of ${ }^{235} \mathrm{U}$ and ${ }^{239} \mathrm{Pu}$. While the errors are not large, the higher order schemes remain more accurate. They also maintain an advantage for most other heavy metals. The largest differences are observed for ${ }^{242 \mathrm{~m}} \mathrm{Am}$ (Fig. 6a), ${ }^{241} \mathrm{Pu}$, and ${ }^{237} \mathrm{U}$ with smaller ones for ${ }^{238} \mathrm{Pu}$ and other americium and curium isotopes.

For ${ }^{242} \mathrm{Pu}$ (Fig 6b) the higher order schemes are actually less accurate than the lower order ones. ORIGEN-style renormalization also makes Ce/CM more accurate than LE for the initial gadolinium, but LE/QI remains the most accurate and $\mathrm{CE} / \mathrm{CM}$ the least accurate. For ${ }^{240} \mathrm{Pu}$, $\mathrm{Ce} / \mathrm{CM}, \mathrm{LE}$, and LE/QI produce comparable results, but $\mathrm{CE} / \mathrm{CM}$ is significantly less accurate. The results for ${ }^{239} \mathrm{U}$ and ${ }^{239} \mathrm{~Np}$ are mixed, and the errors in the concentrations of ${ }^{234} \mathrm{U},{ }^{236} \mathrm{U}$, and ${ }^{238} \mathrm{U}$ are below $0.1 \%$ with all methods.
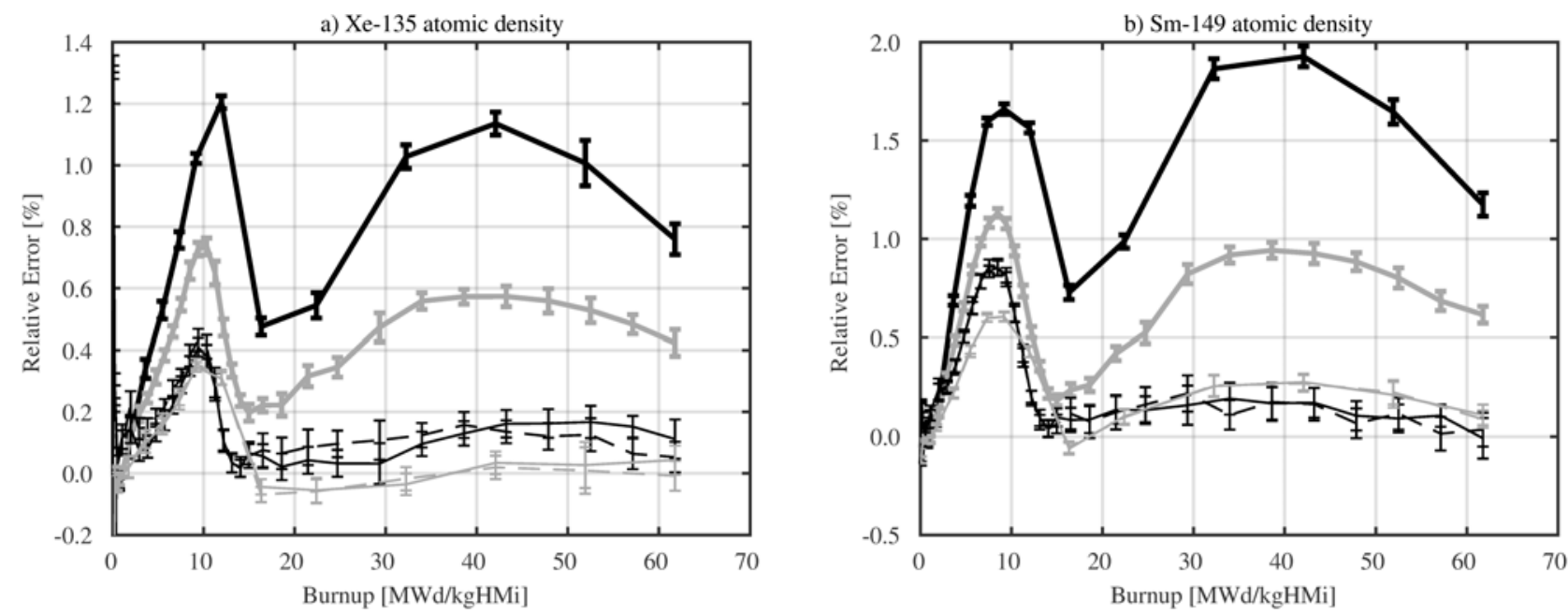

CE/CM-ENE-8

- Ce/CM-ENE-8

LE-NONE-8 - - LE-ENE-8

LE/QI-NONE-8 - - LE/QI-ENE-8

Figure 4: Relative errors in ${ }^{135} \mathrm{Xe}$ and ${ }^{149} \mathrm{Sm}$ atomic densities with different coupling schemes and renormalization strategies. 

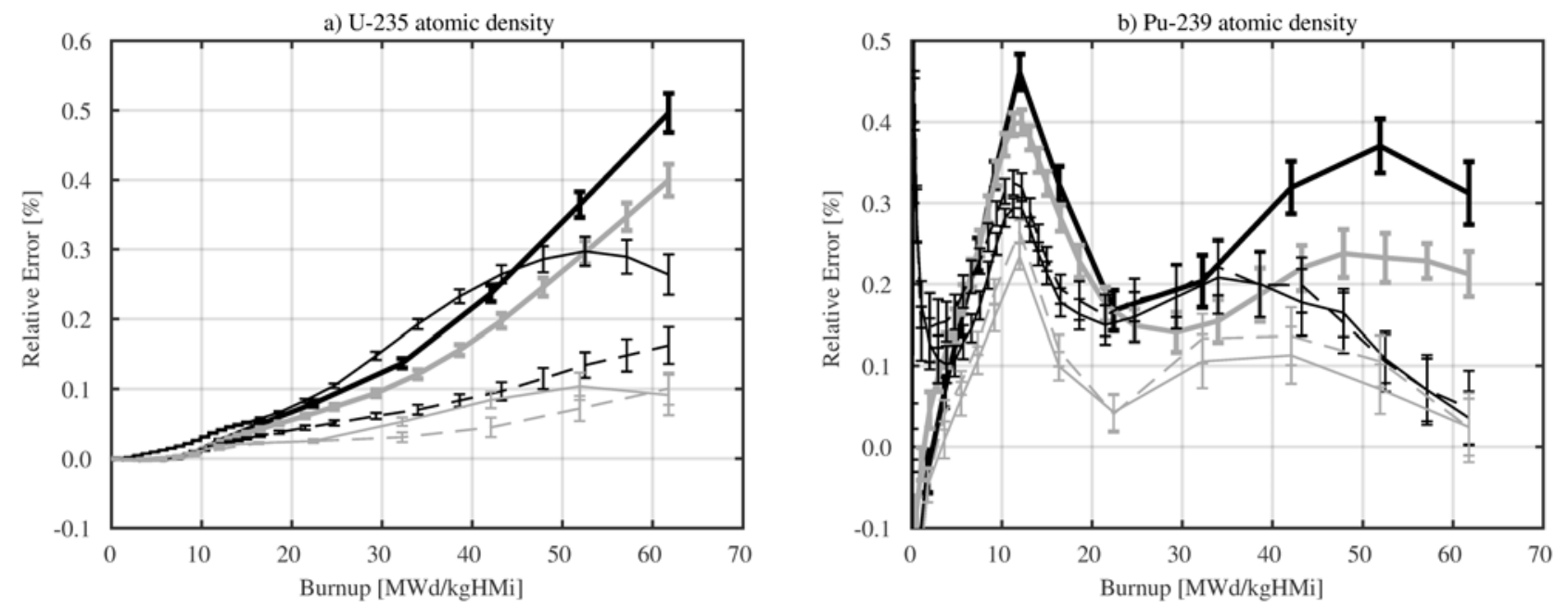

CE/CM-ENE-8 Ce/CM-ENE-8 LE-NONE-8 - - - LE-ENE-8

LE/QI-NONE-8 - - - LE/QI-ENE-8

Figure 5: Relative errors in ${ }^{235} \mathrm{U}$ and ${ }^{239} \mathrm{Pu}$ atomic densities with different coupling schemes and renormalization strategies.
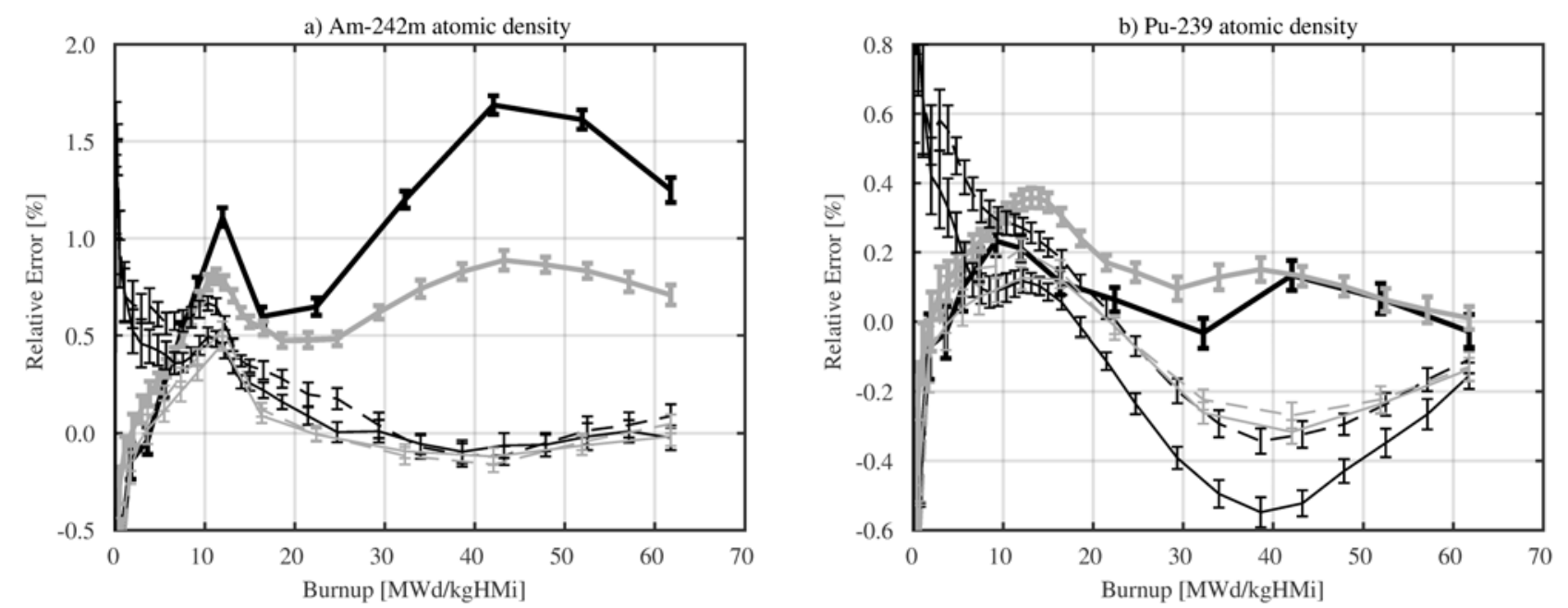

CE/CM-ENE-8 Ce/CM-ENE-8 LE-NONE-8 - - - LE-ENE-8

LE/QI-NONE-8 - - LE/QI-ENE-8

Figure 6: Relative errors in ${ }^{242 \mathrm{~m}} \mathrm{Am}$ and ${ }^{242} \mathrm{Pu}$ atomic densities with different coupling schemes and renormalization strategies. 


\subsection{Renormalization on the predictor}

All the results presented above used the same number of substeps on the predictor and corrector of predictor-corrector methods. However, there is no reason why this would have to be the case. Additional tests were performed with $\mathrm{CE} / \mathrm{CM}$, Ce/CM and LE/QI using energy-based renormalization with eight substeps on the corrector and 1,2,4, or 8 substeps on the predictor. In addition, the calculation was repeated so that renormalization was entirely disabled on the predictor, but eight substeps with renormalization were used on the corrector.

The results for $\mathrm{CE} / \mathrm{CM}$ and $\mathrm{Ce} / \mathrm{CM}$ show no effect from renormalization or the number of substeps on the predictor. As observed by Isotalo and Aarnio (2011b), LE/QI shows minor differences between one and eight substeps on the predictor, but whether renormalization is applied to those substeps again has no effect.

\section{Discussion}

There are two sources of error affected by renormalization. First, depletion calculations require the flux to remain constant while it should be continuously changing to maintain the desired power. Second, it is impossible to select a flux that would exactly match the desired power even in a step-average sense.

The first source of error primarily affects short-lived nuclides, whose concentrations reflect the end of step, rather than step average, reaction rates. The magnitude of these errors depends primarily on how far the flux of the last substep is from the correct end-of-step value. This is approximately proportional to the substep length, i.e., inversely proportional to the number of substeps. Since the number of substeps is the deciding factor, there are no significant differences between the renormalization methods, except that beginning-of-substep renormalization requires twice as many substeps to reduce the error by a given amount. This is because the beginningof-substep flux on the last substep is about twice as far from the end-of-step value as the more representative substep averages in the other method.

The second source of error essentially means that the materials end up at an incorrect burnup. It is most apparent in the concentrations of major fissile isotopes and long-lived fission products. Energy-based renormalization almost completely removed this error even without substeps. Middle-of-substep and ORIGEN-style renormalization are also effective and only need a few substeps. Beginning-of-substep renormalization is far less accurate and can even lead to worse results than not renormalizing at all. This happens because using the beginning-of-substep concentrations for the renormalization on each substep means that the composition earlier in time is systematically overrepresented. The fewer substeps there are the larger this overrepresentation. At the extreme case of only one substep, beginning-of-substep renormalization only ever considers the beginning-of-step concentrations to determine the flux used for the whole step. Clearly this is less representative of the step average than, for example, the middle-of-step concentrations that $\mathrm{CE} / \mathrm{CM}$ and $\mathrm{Ce} / \mathrm{CM}$ would use without renormalization.

Nuclides with half-lives on the order of the step length may exhibit a mixture of the above behaviors. Normalization is also not the only source of error, and results are further complicated by various cancellations of errors occurring with or without renormalization. How the errors for ${ }^{135} \mathrm{Xe}$ increase when renormalizing with $\mathrm{Ce} / \mathrm{CM}$ and $\mathrm{CE} / \mathrm{CM}$ (Fig. 1b) is a good example of this. Since ${ }^{135} \mathrm{Xe}$ is short-lived, its end-of-step concentrations should be determined by the endof-step reaction rates. Without substeps, middle-of-step flux and cross sections are used instead. 
Because of this, the production and removal rates of ${ }^{135} \mathrm{Xe}$ are both significantly underestimated, but these errors happen to cancel out. This cancellation of errors leads to good accuracy when renormalization is not used. When renormalization is used, the fission rate, and hence the xenon production rate, on the last substep is much closer to the correct end-of-step value. However, renormalization does not affect microscopic cross sections, so the middle-of-step cross sections are still used for the whole step. Because of this, the removal rate is improved much less than the production rate. This causes the cancellation of errors that occurs without renormalization to break, which in turn causes the ${ }^{135} \mathrm{Xe}$ concentration to become less accurate despite the flux being more accurate. The same does not happen with the higher order schemes (Fig. 4) because they are able to account for the substep-to-substep changes in the cross sections, which means that the accuracy of the removal is also improved when adding substeps.

In the test case of this work, the flux remains fairly constant until $15 \mathrm{MWd} / \mathrm{kgHM}$ and then increases almost linearly. This behavior can be represented accurately in a step average manner by the middle-of-step fluxes in $\mathrm{CE} / \mathrm{CM}$ and $\mathrm{Ce} / \mathrm{CM}$ or the linear and quadratic fits in LE and LE/QI. Therefore, the normalization errors were relatively small, even without renormalization. The errors for short-lived nuclides reached several percent in the test, but most of them have little effect on neutronics.

The higher order schemes, as well as $\mathrm{CE} / \mathrm{CM}$ and $\mathrm{Ce} / \mathrm{CM}$ with renormalization, produced small errors and could have produced acceptable results with longer steps, at least if the early behavior dominated by gadolinium is not considered. With longer steps, renormalization would have had a larger effect. The effects of renormalization would also be larger in cases where the flux changes faster or in a nonlinear manner. Equivalently, they would also be smaller with shorter steps or slower changing flux.

\subsection{Flux or power distribution}

Renormalization may also affect the results through the spatial distribution of the neutron flux. Renormalization can be performed using whatever flux distribution the coupling scheme predicted, but it is also possible to predict and use the local powers (i.e., the power distribution) instead. In this case, the local fluxes are calculated from the local powers on each substep, causing the flux distribution to change. In this work, the choice was tied to the renormalization method. ORIGEN-style renormalization assumed constant power distribution, while the other methods used flux distribution. However, any of the methods could be implemented based on either flux or power distribution. Renormalization based on local power requires no knowledge of the rest of the system, so each material can be depleted independent of the others. Using the flux distribution requires one global sum per substep, which might affect running times in massively parallel applications.

The only significant difference observed between these options was for the gadolinium absorber, where constant power distribution led to better accuracy. The manual of TRITON (Jessee and DeHart, 2011), which uses constant power renormalization, recommends turning it off for gadolinium rods, so the improvement might not be reliable. In general, which choice leads to better results will likely depend on the problem being modeled.

Choosing between the flux and power distributions might have a more substantial effect in larger geometries, where the distributions are less uniform. On the other hand, the higher order schemes do not require either distribution to be forced constant, so using one or the other should make less of a difference than with the older schemes. 


\subsection{Running times}

Beginning-of-substep renormalization only has to deplete the materials once per substep, which makes it faster than the other methods for a fixed number of substeps. The other methods require the materials to be depleted twice on each substep. Energy-based renormalization also requires the energy released during the first depletion of each substep to be calculated, which makes it slightly slower if the energy release is not already calculated for other purposes. With CRAM, calculating the energy release slows the depletion down by roughly $10 \%$ (Isotalo, 2015b). While fastest on a substep-to-substep basis, the beginning-of-substep renormalization consistently produced less accurate results than the other tested methods and would require more than twice as many substeps to reach given accuracy. This more than negates the advantage it might have in terms of running time and indicates that in practice it cannot compete with the other methods.

Renormalization on the predictors of the tested predictor-corrector coupling schemes had practically no effect. Some other coupling schemes, in particular Ce/BE and CE/BE (Isotalo and Sahlberg, 2015), might still benefit from renormalization and substeps on the predictor. On correctors and in non-predictor-corrector schemes, four substeps were sufficient to gain most of the possible benefits and using more than eight provided only a minor improvement unless using the inaccurate beginning-of-substep renormalization. In the tests of this work, increases in the total running time were lost to noise, but comparing the relative speeds of transport and depletion in individual runs suggests that renormalizing with substeps on the corrector only increased the average running time of $\mathrm{CE} / \mathrm{CM}$ and $\mathrm{LE} / \mathrm{QI}$ by roughly $0.1 \%$ per substep when compared to not using renormalization or substeps. For LE and Ce/CM, which only solve transport once per step, the relative increase is twice as large, but fewer substeps might suffice due to shorter steps. In general, whether renormalization and substeps are worth using will depend on the relative speeds of the depletion and transport calculations, as well as on how accurate the coupling scheme is without them.

\subsection{Renormalization with higher order coupling schemes}

The time dependent fluxes predicted in LE, and especially LE/QI, produce fairly accurate step and substep averages, even without renormalization. Combined with substeps, they also reduce the error from flux discretization in the same way as renormalization with substeps. While middle-of-substep or energy-based renormalization consistently improved the results of LE and LE/QI, the improvement was much smaller than with the older coupling schemes simply because there is less room for improvement.

Isotalo and Sahlberg (2015) and Isotalo (2015a) observed the higher order schemes to have a significant advantage in accuracy over several older methods when renormalization is not used. $\mathrm{CE} / \mathrm{CM}$ and $\mathrm{Ce} / \mathrm{CM}$ were the best performing old methods in those studies. As renormalization benefits the older coupling schemes more than it benefits the higher order ones, this advantage is reduced when renormalization is used. However, the higher order schemes remain more accurate overall than the older ones. This is true even when the higher order schemes do not use renormalization but the older ones do, in which case the higher order methods have slightly larger normalization errors, but their better accuracy in treating cross-sections more than compensates for it. Without renormalization, each substep in the higher order schemes only requires one depletion and no global communication.

Isotalo and Sahlberg (2015) and Isotalo (2015a) used three constant power test cases, only one of which was considered here. Their results for the boiling water reactor (BWR) case were fairly 
similar to those for the PWR case (which is the one used here), while all coupling schemes were accurate in the sodium-cooled fast reactor (SFR) case. Therefore, including those test cases would not likely have affected the conclusions of this study. Note that while LE/LI (Isotalo and Aarnio, 2011a,b) was not included in this paper, it is still considered relevant and might be preferable over LE/QI.

\subsection{Further considerations}

The renormalization methods considered in this work are already so accurate that no room remains for a meaningful increase in accuracy. Instead, to further improve renormalization, running time would need to be reduced without losing accuracy. This would require finding an accurate method to renormalize with just one depletion per substep. One possibility might be to renormalize the flux at the beginning of each substep, but then linearly extrapolate the changes in the local fluxes from the beginning of the previous substep to the middle of the current substep. The first substep within each step would use one of the renormalization methods considered in this work. Only one depletion would be required on each subsequent substep. This approach was not explored because the effect on running time is already small in Shift and because the higher order coupling schemes benefit so little from renormalization.

With the library data used in this work, energy is only directly produced from neutron induced reactions. Decay heat is accounted for implicitly by including it in the energy released from neutron-induced reactions using a secular equilibrium approximation. Energy-based renormalization might be less accurate when decay energy is accounted for explicitly, but the reduction is not expected to be large, as most decay energy is released soon after the preceding reaction, making it effectively proportional to the flux. If needed, the renormalization could be iterated by using the energy released when re-depleting the materials to re-adjust the flux. By repeating this process until convergence, one could guarantee the normalization errors to be below an arbitrary limit.

\section{Conclusions}

A new renormalization method based on calculating the energy released in depletion calculations was proposed and shown to be very accurate. However, even when the renormalization is accurate, substeps are required to reduce the error from the discretization of the flux. When substeps are used, other renormalization methods are comparably accurate.

Renormalization considerably improved the overall accuracy of burnup calculations with two different midstep neutronics-depletion coupling schemes. Similar improvements should occur with other older coupling schemes. Because they are more accurate to begin with, results from the newer higher order coupling schemes are improved less. While using renormalization reduces the differences between different coupling schemes, their order of accuracy is not affected.

Renormalization had no notable effect on the predictors of the tested predictor-corrector coupling schemes. On the corrector and with non-predictor-corrector schemes, four to eight substeps should be sufficient. In general, whether renormalization is worthwhile and the optimal number of substeps will depend on the relative speeds of the depletion and transport calculations, as well as on the accuracy of the coupling scheme.

Renormalization can be based on assuming either the flux distribution or the power distribution to be known. This choice had only a minor effect on the test case of this work and was not 
studied in detail. This choice and renormalization in general might have a bigger effect in other cases, especially when considering larger geometries.

\section{Acknowledgments}

Funding from SAFIR2018, the Finnish Research Program on Nuclear Power Plant Safety, is acknowledged. This material is based upon work supported by the U.S. Department of Energy, Office of Nuclear Energy, Advanced Modeling and Simulation Office, under the Nuclear Energy Advanced Modeling and Simulation (NEAMS) program.

Jessee, M.A., DeHart, M.D., 2011. TRITON: A Multipurpose Transport, Depletion and Sensitivity and Uncertainty Analysis Module, ORNL/TM-2005/39, Version 6.1. Available from Radiation Safety Information Computational Center at Oak Ridge National Laboratory as CCC-785.

El Bakkari, B., El Bardouni, T., Merroun, O., El Younoussi, Ch., Boulaich, Y., Chakir, E., 2009. Development of an MCNP-tally based burnup code and validation through benchmark exercises. Ann. Nucl. Energy. 36, 626-633.

Gauld I.C. 2011. ORIGEN: Depletion Module to Calculate Neutron Activation, Actinide transmutation, Fission Product Generation, and Radiation Source Terms. In SCALE: A Comprehensive Modeling and Simulation Suite for Nuclear Safety Analysis and Design, ORNL/TM-2005/39, Version 6.1. Available from Radiation Safety Information Computational Center at Oak Ridge National Laboratory as CCC-785.

Gauld, I.C., Radulescu, G., Ilas, G., Murphy, B.D., Williams, M.L., Wiarda, D., 2011. Isotopic Depletion and Decay Methods and Analysis Capabilities in SCALE. Nucl. Technol. 174, 169 195.

Gerrity III, T.P., 2012. MCODE-3: Time-dependent Depletion Isotopics with MCNP-5 and SCALE-6.1. Master's thesis, Massachusetts Institute of Technology.

Goorley, T., et al., 2012. Initial MCNP6 Release Overview: MCNP6 Version 1.0. Nucl. Technol. $164,3-12$.

Haeck, W., 2012. VESTA User's Manual Version 2.1.0. DSU/SEC/T/2011-81 - Index A. Institut de Radioprotection et de Sûreté Nucléaire, France

Isotalo, A.E., 2015a. Comparison of Neutronics-Depletion Coupling Schemes for Burnup Calculations - Continued Study. Nucl. Sci. Eng. 180, 286-300. http://dx.doi.org/10.13182/NSE14-92

Isotalo, A.E., 2015b. Calculating Time-Integral Quantities in Depletion Calculations. Submitted to Nucl. Sci. Eng.

Isotalo, A.E., Aarnio, P.A., 2011a. Higher order methods for burnup calculations with Bateman solutions. Ann. Nucl. Energy 38, 1987-1995. http://doi.org/10.1016/j.anucene.2011.04.022

Isotalo, A.E., Aarnio, P.A., 2011b. Substep methods for burnup calculations with Bateman solutions. Ann. Nucl. Energy 38, 2509-2514. http://doi.org/10.1016/j.anucene.2011.07.012 
Isotalo, A.E., Sahlberg, V., 2015. Comparison of Neutronics-Depletion coupling Schemes for Burnup Calculations. Nucl. Sci. Eng. 179, 434-459. http://dx.doi.org/10.13182/NSE14-35

Jessee, M.A., et al., 2014. Polaris: A New Two-dimensional Lattice Physics Analysis Capability for the Scale Code System. PHYSOR 2014, Kyoto, Japan, September 28 - October 3, 2014.

Leppänen, J., 2015. Serpent - a Continuous-energy Monte Carlo Reactor Physics Burnup Calculation Code. VTT Technical Research Centre of Finland (June 18, 2015). www.montecarlo.vtt.fi

Oak Ridge National Laboratory, 2011. SCALE: A Comprehensive Modeling and Simulation Suite for Nuclear Safety Analysis and Design, ORNL/TM-2005/39, Version 6.1. Available from Radiation Safety Information Computational Center at Oak Ridge National Laboratory as CCC785.

Pandya, T.M., Johnson, S.R., Davidson, G.G., Evans, T.M., and Hamilton, S.P., 2015. Shift: A Massively Parallel Monte Carlo Radiation Transport Package, MEC 2015, Nashville, Tennessee, April 19-23, 2015.

Rearden, B.T., Jessee, M.A. Eds., 2016. SCALE Code System, ORNL/TM-2005/39, Version 6.2. Oak Ridge National Laboratory. Available from Radiation Safety Information Computational Center as CCC-834.

Rhodes, J., Smith, K., Lee, D., 2007. CASMO-5/CASMO-5M, a Fuel Assembly Burnup Program, User's Manual. Studsvik Scandpower. SSP-07/431 (2007)

Stankovskiy, A., Van den Eynde G., Vidmar, T., 2010. Development and validation of ALEPH Monte Carlo burn-up code. Nuclear measurements, Evaluations and Applications - NEMEA-6, Krakow, Poland, 25-28 October 2010.

Zhu, A., Collins, B., Kochunas, B., Downar, T., 2014 Assessment of the Depletion Capability in MPACT. PHYSOR 2014, Kyoto, Japan, 28-3 October 2014. 